\title{
Quantitative insights into the dislocation source behavior of twin boundaries suggest a new dislocation source mechanism
}

\author{
Juan Li ${ }^{1,3, a)}\left(\mathbb{C}\right.$, G. M. Pharr ${ }^{2}$, C. Kirchlechner ${ }^{1,3}$ \\ ${ }^{1}$ Max-Planck-Institut Für Eisenforschung GmbH, 40235 Düsseldorf, Germany \\ ${ }^{2}$ Texas A\&M University, College Station, TX 77843, USA \\ ${ }^{3}$ Present Address: Institute for Applied Materials (IAM), Karlsruhe Institute of Technology, 76344 Eggenstein-Leopoldshafen, Germany \\ a) Address all correspondence to this author. e-mail: juan.li@kit.edu \\ Received: 16 February 2021; accepted: 18 May 2021; published online: 4 June 2021
}

George Pharr was an editor of this journal during the review and decision stage. For the JMR policy on review and publication of manuscripts authored by editors, please refer to http://www.mrs.org/editor-manuscripts/.

Pop-in statistics from nanoindentation with spherical indenters are used to determine the stress required to activate dislocation sources in twin boundaries (TBs) in copper and its alloys. The TB source activation stress is smaller than that needed for bulk single crystals, irrespective of the indenter size, dislocation density and stacking fault energy. Because an array of pre-existing Frank partial dislocations is present at a TB, we propose that dislocation emission from the TB occurs by the Frank partials splitting into Shockley partials moving along the TB plane and perfect lattice dislocations, both of which are mobile. The proposed mechanism is supported by recent high resolution transmission electron microscopy images in deformed nanotwinned (NT) metals and may help to explain some of the superior properties of nanotwinned metals (e.g. high strength and good ductility), as well as the process of detwinning by the collective formation and motion of Shockley partial dislocations along TBs.

\section{Introduction}

Grain boundaries and their structures play a vital role in the mechanical properties of polycrystalline materials. For instance, it is well accepted that grain boundaries obstruct dislocation motion and thus enhance the yield strength of most metals and alloys $[1,2]$. Moreover, the important role of grain boundaries in dislocation nucleation and multiplication has been known for some time. For instance, in 1963, J. C. M. Li proposed that dislocations can be generated from grain boundary (GB) ledges, and by assuming that the generated dislocation density is proportional to the unit grain boundary surface area, he found an inverse relation between strength and grain diameter similar to the Hall-Petch relation [3]. There is also experimental evidence that dislocations nucleate in the vicinity of grain boundaries, as observed with transmission electron microscopy (TEM) [4]. Many experimental studies have focused on coherent $\Sigma 3$ twin boundaries (TBs), which are of particular interest because bulk materials with nanotwinned (NT) structures composed of coherent TBs with a spacing of less than $100 \mathrm{~nm}$ [5] often show unique mechanical properties [6-8]. NT materials frequently offer high strength and good ductility - two properties that are typically mutually exclusive.

Today, it is well accepted that defects such as steps and grain boundary dislocations (GBDs) located at $\Sigma 3$ TBs play an important role in the plastic deformation of NT materials [9]. Molecular dynamics simulations have shown that dislocation nucleation from defects at TBs is especially important, and that the high strength of NT materials may result from the large nucleation/ activation stress for these defects [7, 9]. However, experimentally it is very difficult to identify deformation mechanisms in NT materials, since the controlling processes are believed to occur very locally, very quickly, and at very high stresses. In fact, from an experimental perspective, the stress required to activate a grain boundary dislocation source is essentially unknown. With this in mind, the primary aims of this work are to: (i) establish a new technique to measure the stress needed to activate grain 
boundary dislocation sources, and (ii) use the new technique to explore the intrinsic source activation strength of coherent $\Sigma 3$ twin boundaries in copper $(\mathrm{Cu})$ and copper-aluminum $(\mathrm{Cu}-$ $\mathrm{Al})$ alloys.

\section{Theory}

The technique we propose involves measurements of the pop-in stress when spherical nanoindentations are made near a twin boundary. Pop-in is well known to correlate with the onset of plastic deformation during spherical nanoindentation [10-13]. During spherical nanoindentation, a purely elastic stress field first develops beneath the indenter, as described by Hertzian contact mechanics (see Fig. 1a). The maximum shear stress $\tau_{\text {max }}$ in that region depends on the indenter radius, $R$, the reduced elastic modulus of the sample-indenter system, $E_{r}$, and the load on the indenter, $P$, through [10]:

$$
\tau_{\max }=0.31\left(\frac{6 P E_{r}^{2}}{\pi^{3} R^{2}}\right)^{1 / 3},
$$

where $E_{r}$ is the reduced elastic modulus given by

$$
\frac{1}{E_{r}}=\frac{1-v_{s}^{2}}{E_{s}}+\frac{1-v_{i}^{2}}{E_{i}} .
$$

$E_{s}$ and $E_{i}$ are the elastic moduli of the sample and the indenter; $v_{s}$ and $v_{i}$ are the Poisson ratio for the sample and the indenter, respectively.

The maximum shear stress occurs at a specific depth beneath the sample surface, and the contact is characterized by a reversible, non-linear load versus indenter-displacement curve (see Fig. 1b). As the load on the indenter increases, the maximum shear stress, $\tau_{\max }$, as well as the volume which is under high levels of stress, increases until either the shear stress reaches the theoretical strength or the highly stressed volume samples a pre-existing dislocation source. In both cases, a sudden elastic-plastic transition occurs characterized by an abrupt displacement burst which produces a "pop-in" in the load versus displacement curve (see horizontal line in Fig. 1b). The load at pop-in, $P_{p o p-i n}$, can be used to calculate the maximum shear stress beneath the indenter according to Eq. (1), which is further interpreted as the dislocation nucleation or source activation stress $[10,12]$. The use of pop-in statistics to probe the strength distribution of single crystals at the micrometre length scale is now well established $[10,14-16]$. The technique is also used to probe the theoretical strength of metals $[14,17,18]$ and study the mechanisms of hydrogen embrittlement [19].

In this study, the benefits of spherical indentation with pop-in statistics are that it can be performed with a statistically significant number of experimental observations and that the technique is site-specific, that is, it can be localized very near a grain or twin boundary. This is achieved by making spherical nanoindents either at coherent $\Sigma 3$ twin boundaries or in the grain interior and compare the underlying pop-in-statistics.

\section{Results}

Polycrystalline $\mathrm{Cu}$ was utilized for nanoindentation testing $[17,20]$. All experiments and examinations were performed on (110)-oriented grains and TBs, since TBs in this orientation are perpendicular to the surface. Before indenting, the quality of the electropolished surface near and away from the TB was examined by atomic force microscopy (AFM, Veeco Dimension 3100). The area near the TB had the same roughness as the grain interior, and there were no additional surface steps at the TB (see (a)

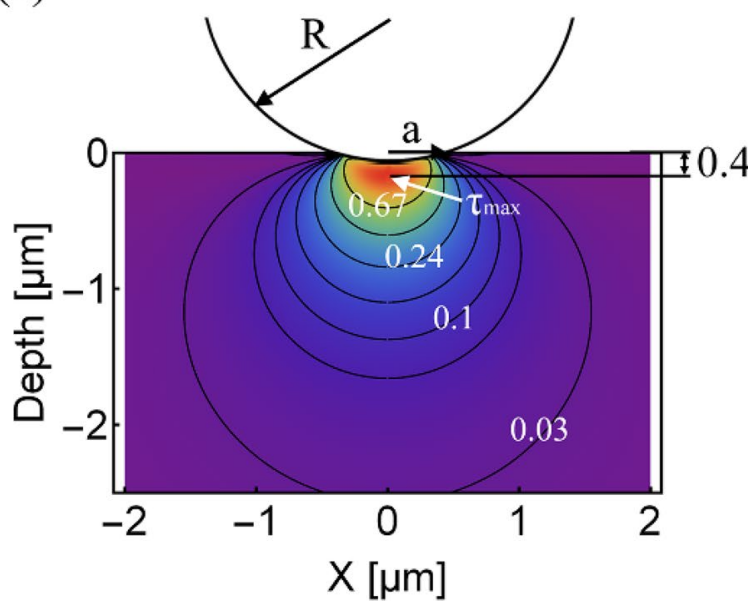

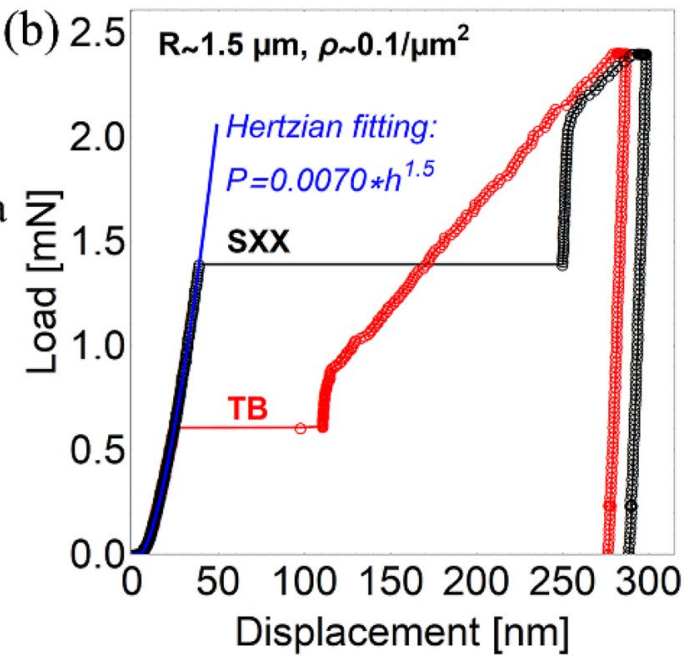

Figure 1: (a) Stress distributions beneath a spherical indenter. The local shear stress is normalized to the maximum shear stress $\tau_{\text {max }}$. (b) Representative load-displacement curves at the TB and in the single crystal (SXX) reference. 
supplementary Fig. S1). Nanoindentation was performed with an Agilent G200 Nanoindenter (Agilent Technologies) using a spherical diamond indenter with a radius of $\sim 1.5 \mu \mathrm{m}$. During indentation, regions showing steps and kinks in the TB as visible in the SEM were deliberately avoided. A line of indents inclined by $1^{\circ}$ with respect to the TB trace at the surface was used to precisely position indents at various known distances from the boundary. In this way, some indents exhibited a contact radius $a$ (see supplementary Eq. S1.1) at the pop-in force larger than the distance of the indent to the TB, thus assuring that the highly stressed volume beneath the indenter intersected the TB. Such indents are denoted here as indents at the TB. Additional indents were placed inside the grains, denoted as SXX.

\section{Differences of pop-in stress}

The pop-in behavior in the interior of single grains (i.e., single crystals) and at twin boundaries is remarkably different. This can be seen from the cumulative probability plots in Fig. 2a of pop-in stresses in single crystals ( 900 indents) and at one TB (41 indents). Two important features are evident. First, the mean pop-in stress at the TB is significantly smaller than in the single crystals, indicating that the onset of plasticity at the boundary occurs at lower stresses. Second, the width of the distribution of the pop-in stresses at the TB is narrower than in the single crystals. To further explore whether the two distribution functions are related to each other, the ratio of the two quantiles are plotted in Fig. 2b (see supplementary file for details of quantile difference plot in Sect. 2). Curiously, the ratio of the pop-in stress at the TB and in a single crystal is constant over the entire distribution width at a value of $0.78 \pm 0.01$. This strongly suggests a link of the two distribution functions.

The reduction of dislocation activation or nucleation stress at the TBs measured by spherical nanoindentation is different from earlier work (e.g. by Britton et al. [21]), which was used to analyze the dislocation transmission behavior of dislocations through a grain boundary.

\section{Microstructure characterization}

Since we can exclude any influence of surface steps (Fig. S1), oxide layers [22], elastic anisotropy and heterogeneity [23] by accompanying measurements presented in Sect. 3 of the supplementary material, the local defect structure at the TB is likely the origin of the vastly different behavior. To explore this, we analyzed the microstructure of the TB by electron backscatter imaging (BSE) in a scanning electron microscope (SEM, Zeiss Gemini 500) and transmission electron microscopy (TEM, JEOL JEM 2200FS). Except for nano-scaled steps visible in the SEM (Fig. 3), Frank partial dislocations were the only frequently observed defect at the TB (see Fig. 4a), and we found no evidence of lattice dislocations or Shockley partial dislocations at the TB. The Frank partial dislocations have a Burgers vector normal to the $(111)_{1}$ TB plane, as determined using the invisibility criterion of $g \cdot \underline{b}=0$ ( $g$ is the diffracting vector and $\underline{b}$ is the Burgers vector).

Frank partial dislocations are known to be present at TBs produced by annealing and are considered as intrinsic defects of TBs $[24,25]$. Our TB shows an array of uniformly distributed parallel Frank partial dislocations with a mean spacing of $\sim 30 \mathrm{~nm}$, which results in a deviation from the perfect $\Sigma 3$ coincident site lattice (CSL) of about $0.49^{\circ}$ (see Fig. 4a).

\section{Results and discussion}

\section{The role of Frank partial dislocations during dislocation multiplication}

It is known that Frank partial dislocations can act as dislocation sources in face-centered cubic single crystals [26-29] according to the dislocation reaction:

$$
\frac{a_{0}}{3}[111]_{\text {sessile }}+\frac{a_{0}}{6}[11 \overline{2}]_{\text {glissile }} \rightarrow \frac{a_{0}}{2}[110]_{\text {glissile }}
$$
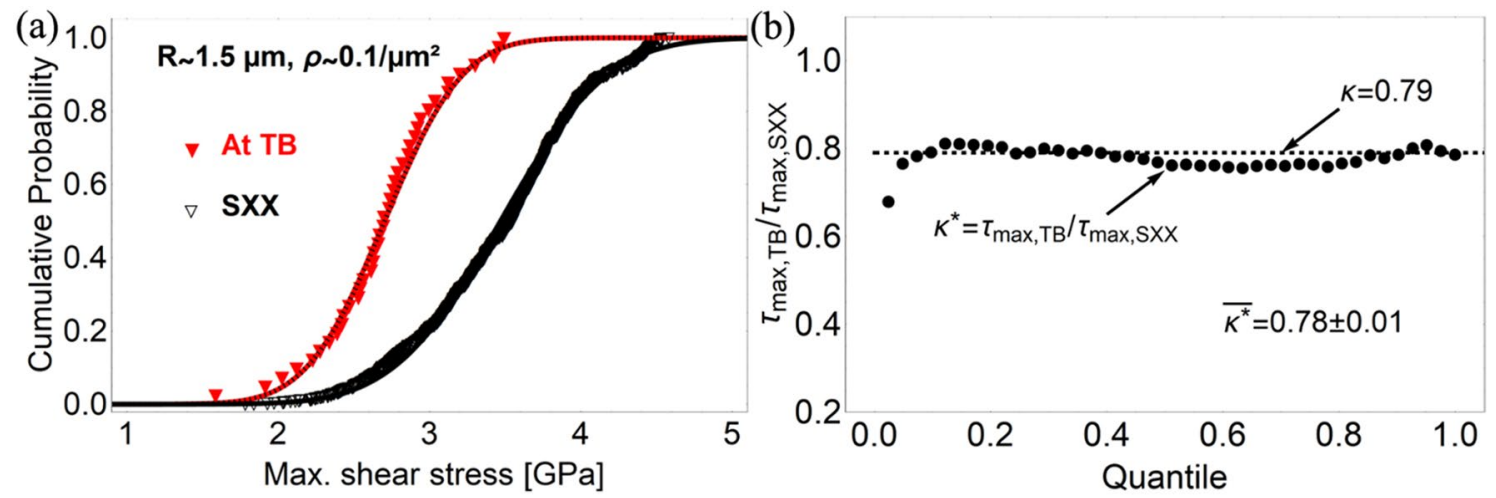

Figure 2: Pop-in statistics at a TB in pure Cu and in the single crystal (SXX) reference. (a) Cumulative distribution plot. (b) Quantile ratio plot. 


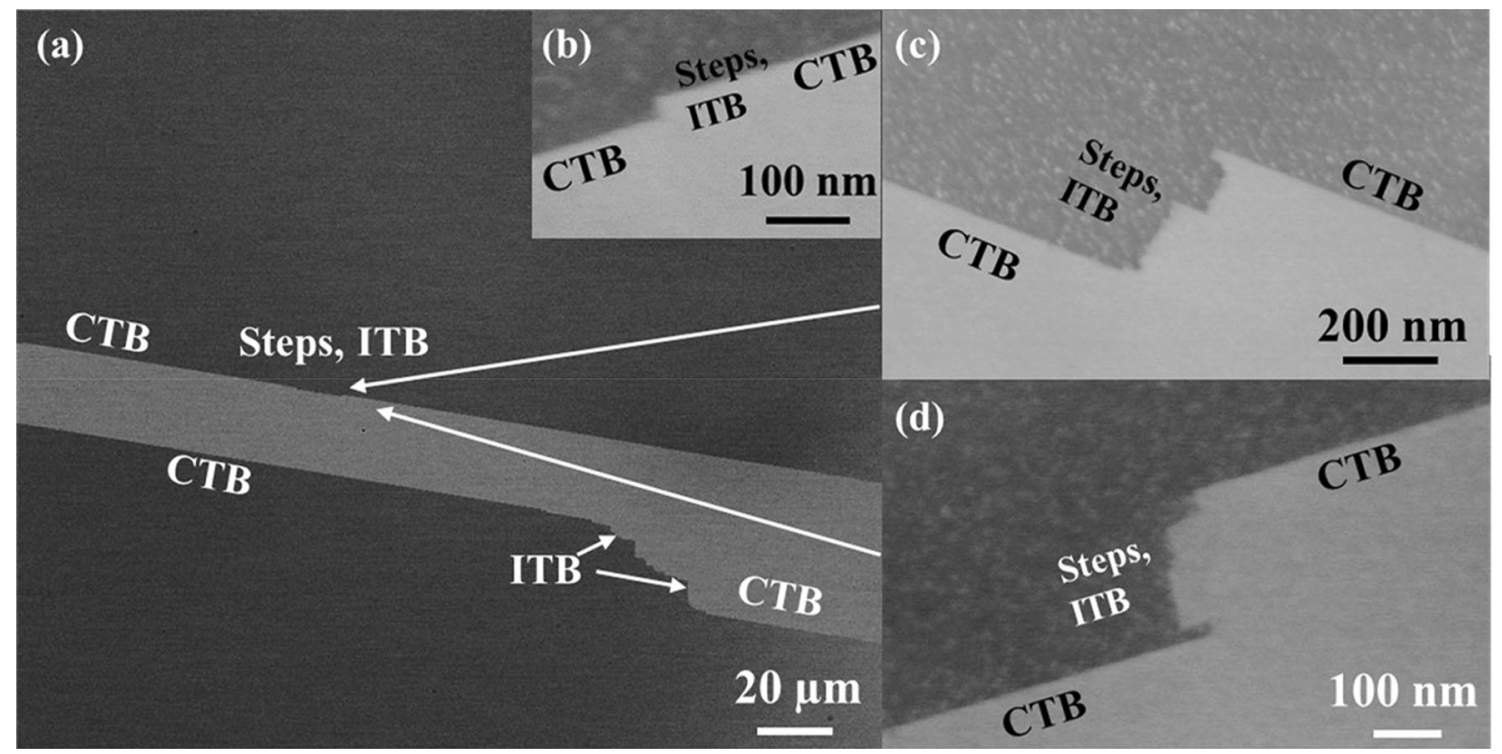

Figure 3: Electron Channeling Contrast Images of an annealed copper twin boundary. (a) Overview. (b,c,d) Magnified views on incoherent steps at the twin boundary.
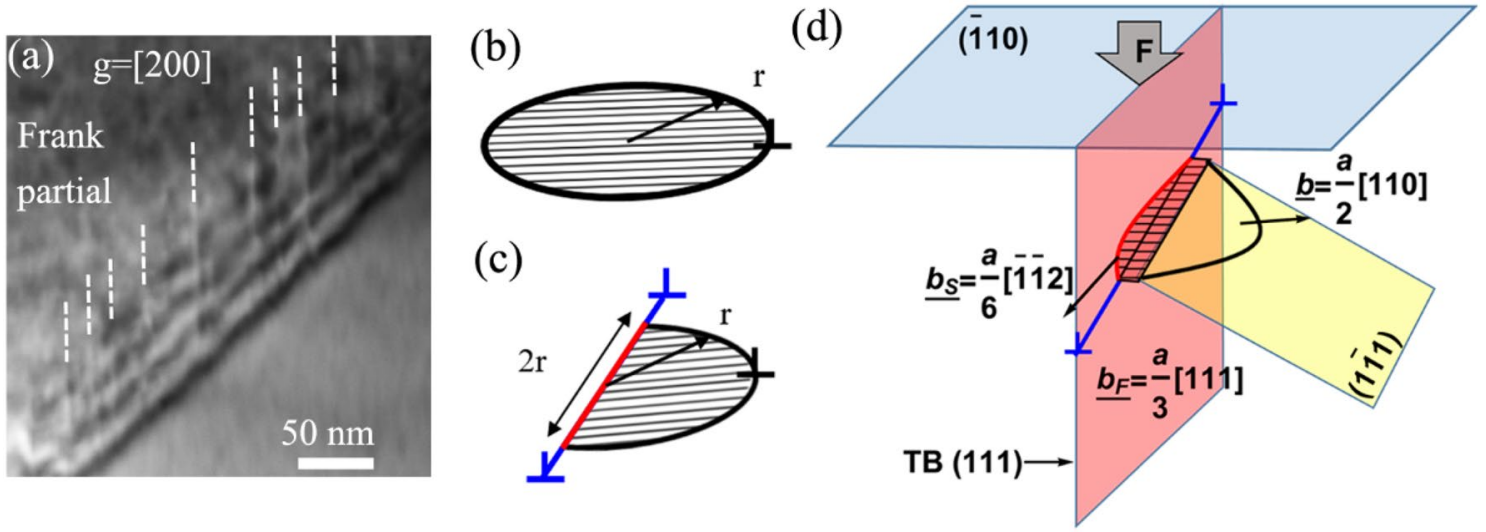

Figure 4: (a) TEM bright field image ( $g=[200]$ ) reveals an array of Frank partial dislocations with a spacing of $\approx 30 \mathrm{~nm}$ at the TB. Schematic of the dislocation nucleation in (b) a single crystal and (c) at a Frank partial dislocation in the TB plane. (d) Schematic of the Frank partial dislocation during dissociation into a perfect dislocation half loop in the $(1 \overline{1} 1)$ plane and a Shockley partial dislocation in the (111) plane.

in which a pre-existing sessile Frank partial dislocation interacts with a gliding Shockley partial to form a glissile lattice dislocation.

In materials with high stacking fault energy (SFE), like $\mathrm{Al}$, this reaction occurs spontaneously [28]. However, for low SFE materials, additional local stress or thermal activation is required [29]. We note that other Frank partial dissociation reactions that result in glissile lattice dislocations and mobile Shockley partial dislocations have been observed but such Frank partial dissociation reactions are not discussed here [26, 27, 29].

Based on this, we hypothesize that the pre-existing Frank partial network at the TB plays an important role in the mechanical behavior of the TB and produces a dislocation activation stress different from single crystals. This is because a Frank partial dislocation can also dissociate into a Shockley partial dislocation and a full lattice dislocation according to

$$
\frac{a_{0}}{3}[111]_{\text {sessile }} \rightarrow \frac{a_{0}}{6}[\overline{112}]_{\text {glissile }}+\frac{a_{0}}{2}[110]_{\text {glissile }},
$$

both of which are glissile. However, in contrast to the reaction of Eq. (3), the total strain energy of this dislocation reaction increases, and it will therefore only occur in the presence of external stress. Our hypothesis is further supported by recent post mortem high resolution TEM images of L. Lu et al. [6], which essentially shows the dislocation products on the right side of Eq. (4) with a $\frac{a_{0}}{2}[110]_{\text {glissile }}$ dislocation being split into two Shockley partials. 
To quantitatively predict the required stress for a dislocation reaction according to Eq. (4), we consider an expanding dislocation loop of radius $r$, such as that schematically shown in Fig. $4 \mathrm{~b}$. First, we consider a perfect lattice dislocation loop inside a single grain (see Fig. 4b). Upon expansion of the loop, the energy $U$ of the system increases due to the dislocation line energy $\left(G b^{2} / 2\right.$ per unit length with $G$ being the shear modulus) and decreases due to the work done by the dislocation under the applied stress, $\tau$, according to [30]

$$
U=2 \pi r \frac{G b^{2}}{2}-\tau r^{2} \pi b
$$

The loop will spontaneously expand when the total loop energy decreases with further loop expansion, i.e., when $\partial U / \partial r=0$. In this way, the shear stress $\tau_{S X X}$ required for homogeneous nucleation of a lattice dislocation in single crystal can be estimated as

$$
\tau_{S X X}=\frac{G b}{2 r} .
$$

However, according to Eq. (4), the dissociation of a Frank partial dislocation requires two additional energy considerations. First, only a half loop can expand as the lattice dislocation is confined to one grain (see Fig. 4c). Second, the dissociation of the Frank partial involves the deletion of the Frank partial dislocation (Burgers vector $b_{F}$ ) and the formation of a Shockley partial dislocation (Burgers vector $b_{S}$ ) along the former Frank partial dislocation line (see Fig. 4d). For the dissociation of the Frank partial dislocation and half loop formation of a perfect lattice dislocation, the energy of formation is then given by

$$
U=\pi r \frac{G b^{2}}{2}-1 / 2 \tau r^{2} \pi b+2 r\left(\frac{G b_{S}^{2}}{2}-\frac{G b_{F}^{2}}{2}\right) .
$$

For the energetics in Eq. (7), the loop will spontaneously expand $(\partial U / \partial r=0)$ at a shear stress $\tau_{T B}$ given by

$$
\tau_{T B}=\frac{G}{2 r} \frac{\left(\pi b^{2}+2 b_{S}^{2}-2 b_{F}^{2}\right)}{\pi b} .
$$

To relate the dislocation source activation strengths in single crystals and at the TB, we compare Eqs. (6) and (8) and define a ratio $\kappa$, where

$$
\kappa=\frac{\tau_{T B}}{\tau_{S X X}}=1+\frac{2 b_{S}^{2}-2 b_{F}^{2}}{\pi b^{2}} .
$$

Clearly, this ratio only depends on the magnitudes of the Burgers vectors of the lattice dislocation, $b$, the Shockley partial dislocation, $b_{s}$, and the Frank partial dislocation, $b_{P}$ where $|b|=a_{0} / \sqrt{2},\left|b_{S}\right|=a_{0} / \sqrt{6}$ and $\left|b_{F}\right|=a_{0} / \sqrt{3}$. Therefore, for face-centered cubic materials, the ratio of dislocation nucleation involving the dissociation of a Frank partial dislocation at a twin boundary with respect to the perfect dislocation nucleation in the grain interior should be $\kappa=0.79$. Because this process of dislocation activation involves the dissociation of Frank partial dislocations, we refer to it as the "inverse Frank mechanism".

The ratio $\kappa$, i.e., the ratio of the stress required assuming the inverse Frank mechanism and for dislocation nucleation in a single crystal, fits remarkably well to our experimental data (compare $\kappa=0.79$ and $\overline{\kappa^{*}}=0.78 \pm 0.01$ as shown in Fig. 2b). However, the presence of at least one Frank partial dislocation in the highly stressed region beneath the indenter is a pre-condition for the mechanisms being proposed. Referring to the experimental data in Fig. $2 \mathrm{a}$ and noting that $R=1.5 \mu \mathrm{m}$ and $\rho=0.1 /$ $\mu \mathrm{m}^{2}$, the contact radius is of the order of $\sim 100 \mathrm{~nm}$. Similar to Morris et al. [10], we suggest that the highly stressed volume is $\sim a^{3}$ and experiences a shear stress of at least $\sim 1.6 \mathrm{GPa}$ at popin in our case. Based on a Frank partial dislocation distance of $30 \mathrm{~nm}$, we argue that at least one Frank partial dislocation is always present at the TB in this highly stressed volume, and all requirements for the proposed mechanism are fulfilled.

The excellent mechanical properties of NT systems are often attributed to the presence of incoherent twin boundaries (ITBs) and Shockley partial dislocations [6, 9, 31, 32], since they are mobile compared to the Frank partial dislocations. The proposed dissociation of Frank partials, which often are located at ITBs [33], results in a mobile Shockley partial at the TB and a possible dissolution of ITBs.

\section{Verification of the inverse Frank mechanism}

To further examine the proposed mechanism, we conducted additional case studies by varying: (i) the indenter radius, (ii) the dislocation density, and (iii) the SFE. In the first two cases, we changed the pop-in behavior sampling over different dislocation source sizes and dislocation source spacing, which alters the pop-in stress at TBs and in the single crystals (see Table 1 for the indenter radii and dislocation densities used in the experiments). The cumulative probability plots and the ratio of the two quantiles are given in the supplementary Fig. S2. The experimentally observed $\kappa^{*}$ ratio describing the mean pop-in stress at the TB and the mean single crystal pop-in stress remained close to the value for the inverse Frank mechanism (compare (i) $\overline{\kappa^{*}}=0.76 \pm 0.02$; (ii) $\overline{\kappa^{*}}=0.82 \pm 0.01$ ). We also changed the SFE by the addition of $\mathrm{Al}$ to the otherwise pure $\mathrm{Cu}$ and observed a systematic decrease of the pop-in stress in the single crystals (similar to [22]). However, we also found that the pop-in stress at the TB was reduced by a proportional amount, once again resulting in a constant $\overline{\kappa^{*}}$ ratio close to 0.79 as can be seen in Fig. 5. The results show the changes of the $\overline{\kappa^{*}}$ ratio with pre-existing dislocation density, indenter radius and $\mathrm{Al}$ content, as also summarized in Table 1 and shown in Fig. 6. From them, we conclude that irrespective of the dislocation density, indenter radii and SFE, a constant $\overline{\kappa^{*}}$ ratio exists, and this 
TABLE 1: Summary of the pop-in stress in the TB and the SXX for different samples.

\begin{tabular}{ccccccc}
\hline \hline Sample & Al (at\%) & $P\left(10^{-12} \mathrm{~m}^{2}\right)$ & $\begin{array}{l}\text { Indenter } \\
\text { radius }[\mu \mathrm{m}]\end{array}$ & $\overline{\tau_{\max , T B}}[\mathrm{GPa}]$ & $\overline{\tau_{\max , S x x}}[\mathrm{GPa}]$ & $\overline{\kappa^{*}}[1]$ \\
\hline$\# 1$ & 0 & $\sim 0.1$ & 1.5 & $2.70 \pm 0.41$ & $3.47 \pm 0.56$ & $0.78 \pm 0.01$ \\
$\# 2$ & 0 & $\sim 0.1$ & 1 & $3.43 \pm 0.60$ & $4.21 \pm 0.67$ & $0.82 \pm 0.01$ \\
$\# 3$ & 0 & $\sim 2.0$ & 1 & $2.27 \pm 0.33$ & $3.00 \pm 0.51$ & $0.76 \pm 0.02$ \\
$\# 4$ & 0 & $\sim 0.06$ & 1 & $3.10 \pm 0.35$ & $3.88 \pm 0.61$ & $0.80 \pm 0.03$ \\
$\# 5$ & 3 & $\sim 0.06$ & 1 & $2.66 \pm 0.29$ & $3.32 \pm 0.46$ & $0.80 \pm 0.02$ \\
$\# 6$ & 4 & $\sim 0.06$ & 1 & $2.42 \pm 0.37$ & $3.20 \pm 0.36$ & $0.76 \pm 0.03$ \\
$\# 7$ & 6 & $\sim 0.06$ & 1 & $2.24 \pm 0.49$ & $2.91 \pm 0.46$ & $0.77 \pm 0.05$ \\
\hline \hline
\end{tabular}

compares remarkably well to the ratio predicted for the inverse Frank mechanism.

The cumulative probability (CP) distribution of some specimens in the grain interior and at TBs cannot be fitted by a symmetric distribution (see for instance Fig. 5e and Fig. S2c). This phenomenon and a quantitative model is well described for molybdenum single crystals with different dislocation density and indenter radii [18]: a small or large product $R \rho_{\text {def }}^{1 / 3}$, with $\rho_{\text {def }}$ being the dislocation density and $\mathrm{R}$ as the indenter radius, results in a symmetric pop-in distribution, while an intermediate value of $R \rho_{d e f}^{1 / 3}$ will lead to a wider and bi-modal distribution of the pop-in stress. In this case, the CP indicates a bi-modal (asymmetric) distribution because the highly stressed volume either contains pre-existing dislocations or does not contain preexisting dislocations. The horizontal quantile plot of the ratio of the pop-in stress at TBs to that in grain interior in the entire $\mathrm{CP}$ plot (see Fig. 2b) indicates that Frank partial dislocations, show the same trend; however, a sound statistical analysis of the dislocation structure at the TBs is beyond the scope of this work.

\section{Some general implications}

Finally, we wish to comment on the general implications of the proposed inverse Frank mechanism on the deformation of nanotwinned (NT) materials. Mechanically, NT materials exhibit unusually high strength and tensile ductility - two properties which are mutually exclusive in most engineering materials $[6,8,9]$. During deformation, the microstructure of NT materials is subjected to significant changes, and sometimes the loss of twin boundaries, a process referred to as detwinning $[7,9,34,35]$. The proposed inverse Frank mechanism can potentially explain all three of these observations. The high strength is the relatively easy to understand since, in absence of other dislocation sources, the stress is dominated by the inverse Frank mechanism which requires high stresses. As for the increase in ductility, material failure in tension tests sets in when the intrinsic material hardening behavior cannot compete with local geometrical softening phenomena, as reflected in Considere's criterion [36]. At the macroscopic length scale, localization of deformation (i.e., necking) occurs when the hardening rate falls below the geometric softening rate. At the micron scale, the sources of localization are often very different, involving, for instance, the shear failure of a precipitate or dislocation slip transmission through a grain boundary. The origin of this localization is a combination of a stable dislocation source somewhere in the microstructure and the loss of a strengthening mechanism (e.g., particle failure) due to local stress amplification or dislocation pile-up. In this context, it is notable that the inverse Frank mechanism does not form a stable dislocation source, as the source is destroyed during the emission of the first lattice dislocation. Further plasticity thus requires the activation of a new inverse Frank partial dislocation source. Therefore, the inverse Frank mechanism cannot produce a strong dislocation pile-up and thus prevents localization by this mechanism at the microstructure length scale. As a consequence, the activation of ever new lattice dislocations via the inverse Frank mechanism causes a high stress plateau and increases the strain to failure in NT materials $[6,8,9]$.

Detwinning may also be a natural consequence of the inverse Frank partial mechanism. This is because the perfect lattice dislocation and the Shockley partial produced by the mechanism are both mobile. The Shockley partial dislocations can travel parallel to the TB. In case of collective motion of identical Shockley partial dislocations parallel to a TB, detwinning is expected (see, e.g. [34]). In this regard, it is notable that identical Shockley partial dislocations have been observed in post-deformed NT materials [6].

Lastly, particularly MD simulations showed the ability of grain boundaries to act as source or sink for mobile dislocations in nanocrystalline materials [37-39]. From an experimental viewpoint the quantitative investigation of the dislocation source behavior remains challenging. The presented approach provides a fast tool to experimentally assess the dislocation source behavior of grain boundaries and interfaces.

\section{Conclusion}

In summary, we investigated the pop-in behavior during spherical indentation at TBs and in single crystalline $\mathrm{Cu}$ and $\mathrm{Cu}-\mathrm{Al}$ alloys. We found that the pop-in stress at the TB is always 
(a) 1 .
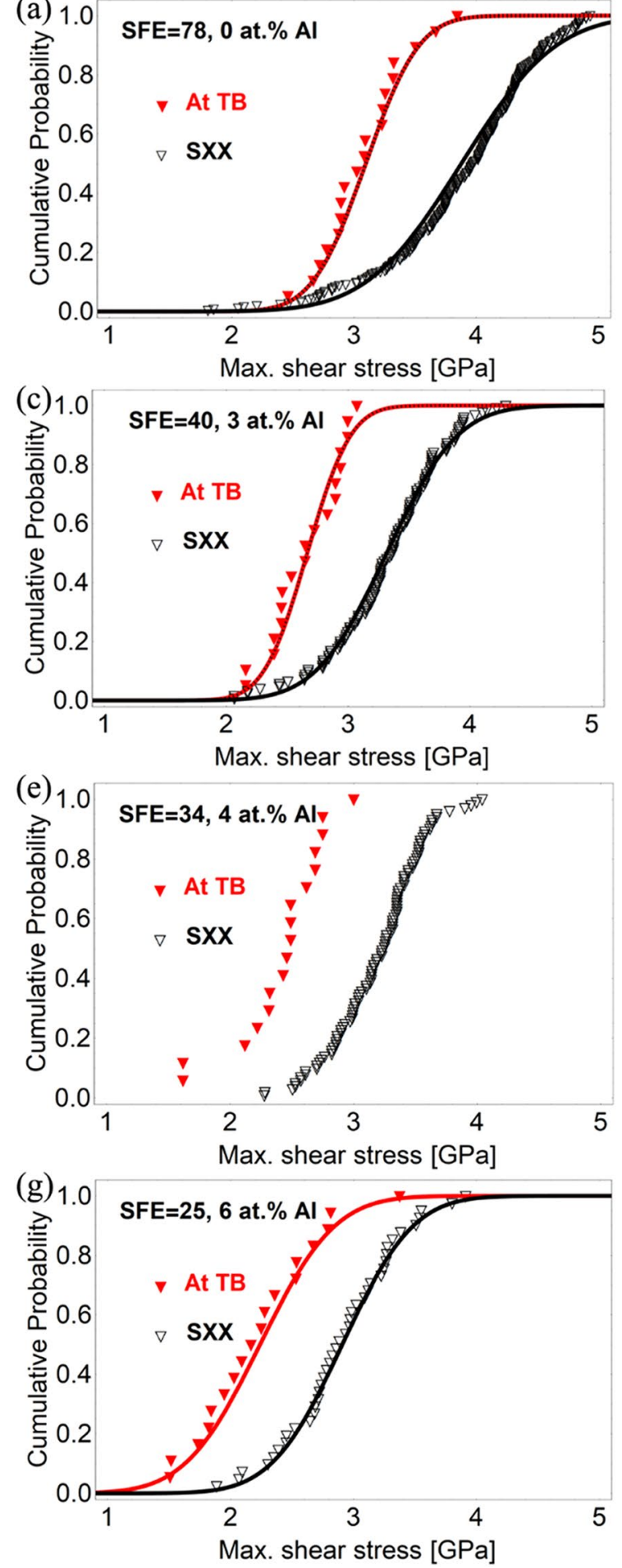

(b)

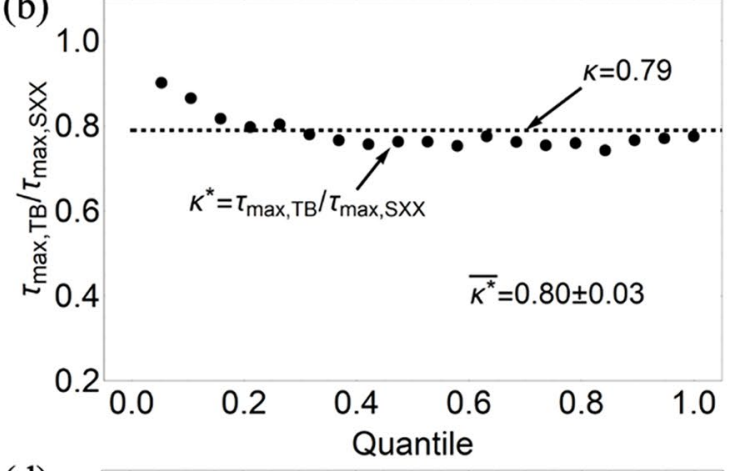

(d)
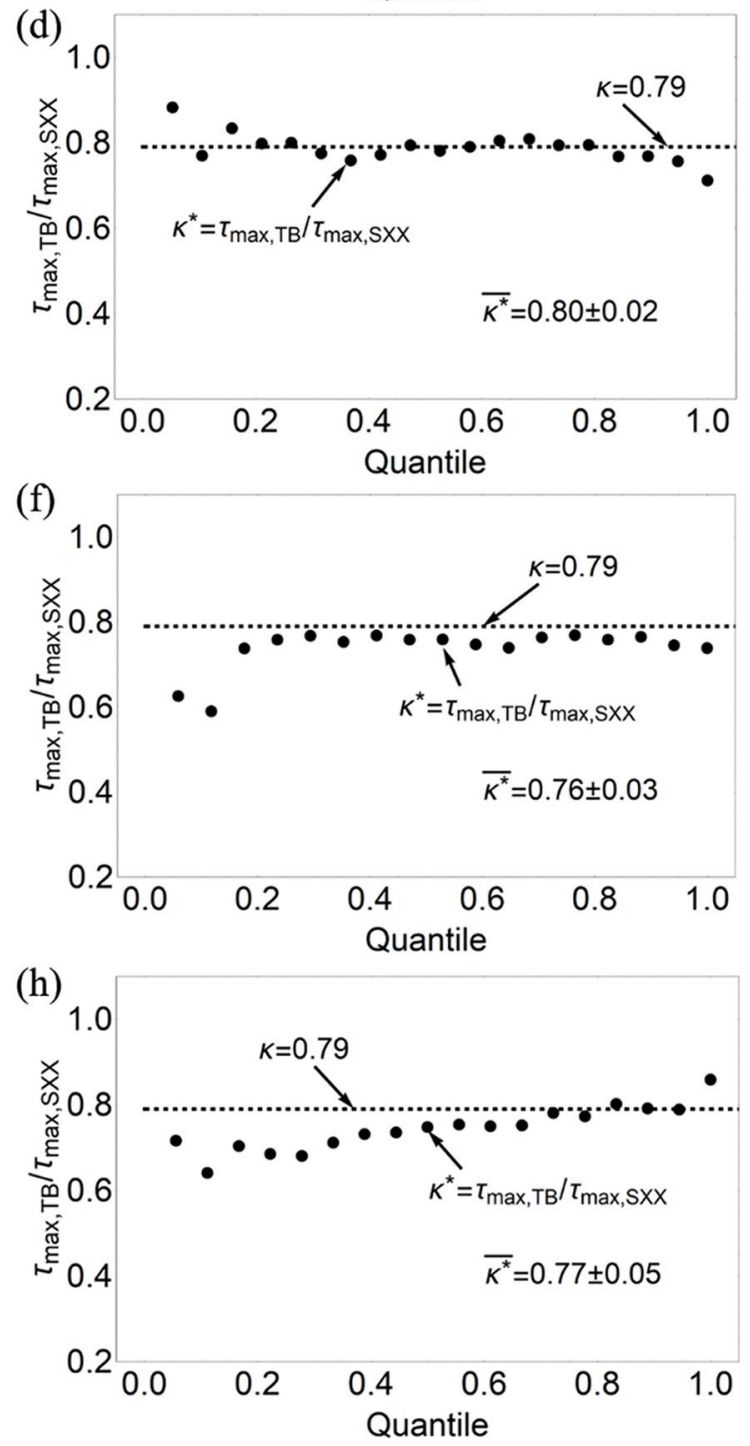

Figure 5: Pop-in statistics at TBs and in SXX in Cu-Al alloy with various SFE.

smaller than in the single crystal, irrespective of the initial dislocation density in the grain, the indenter radius and the SFE. The observed ratio $\overline{\kappa^{*}}$ of the mean TB pop-in stress to the single crystal pop-in stress ranges from 0.76 to 0.82 . To explain this behavior, we propose a dislocation dissociation process denoted as the inverse Frank mechanism in which a pre-existing Frank partial dislocation in the TB dissociates into a mobile Shockley partial in the TB plane and a mobile lattice dislocation. Recent high resolution TEM experiments show all products of the proposed mechanism. As outlined, the inverse Frank mechanism 


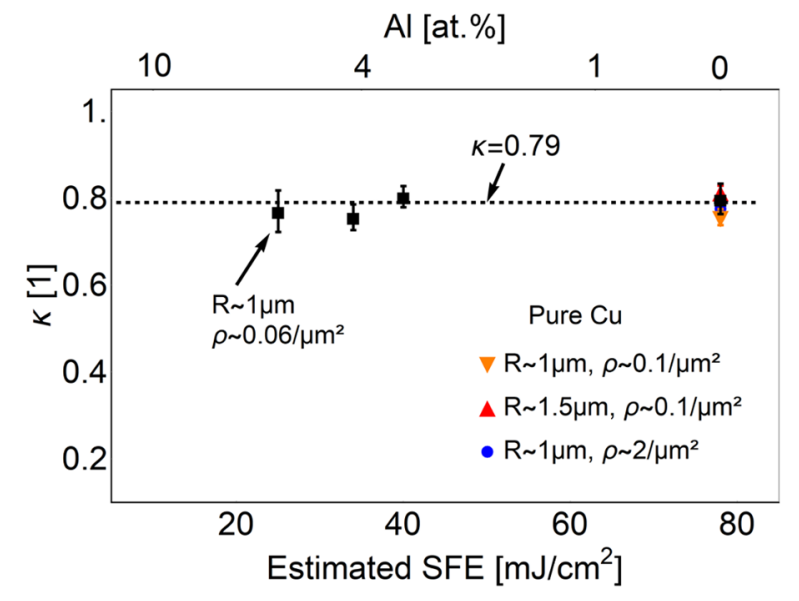

Figure 6: Ratio $\overline{\kappa^{*}}$ comparing the mean of pop-in stresses at the TB and in the SXX for different dislocation densities, indenter sizes and stacking fault energies. The Al content is used to estimate the SFE based on reference [19]. The dashed line $(\kappa=0.79)$ is the geometric prediction from the proposed inverse Frank mechanism.

can explain not only the high strength of NT materials but also possibly their enhanced ductility and detwinning behavior.

\section{Materials and methods}

$\Sigma 3$ twin boundaries were achieved by annealing $\mathrm{Cu}$ and $\mathrm{Cu}-\mathrm{Al}$ alloys. The as-received polycrystalline $\mathrm{Cu}$ (purity 99.99\%) provided by Goodfellow was cold-rolled to a thickness reduction of $70 \%$ with a final thickness of $3 \mathrm{~mm}$. Small pieces $10 \times 10 \mathrm{~mm}^{2}$ were cut by electrical discharge machining (EDM). To control dislocation densities and grain size, two annealing conditions were employed: $950{ }^{\circ} \mathrm{C}$ for $12 \mathrm{~h}$ and $750{ }^{\circ} \mathrm{C}$ for $72 \mathrm{~h} \mathrm{[20].} \mathrm{After}$ grinding with $\mathrm{SiC}$ paper down to a 2400 grit, the samples were electropolished with the electrolyte D2 (Struers, Germany) at $24 \mathrm{~V}$ for $100 \mathrm{~s}$ [17]. The crystallographic orientation was identified by electron backscatter diffraction (EBSD), which also showed that the grain size of each sample is of the order of millimeters.

A diffusion couple with a continuous change of the Al content from 0 at. $\%$ to 20 at.\% was fabricated by heating the bonded samples of a Cu-20 at.\% $\mathrm{Al}$ alloy and pure $\mathrm{Cu}$ at $950{ }^{\circ} \mathrm{C}$ for $48 \mathrm{~h}$. After grinding with $\mathrm{SiC}$ paper down to a 2400 grit, the specimens were polished successively by $3 \mu \mathrm{m}, 1 \mu \mathrm{m}$ and $0.25 \mu \mathrm{m}$ diamond suspension with lubricant from Struers. After each step, the polished surface was checked by optical microscopy, and no larger scratches from last step were observed. To decrease the etching rate in the region with low $\mathrm{Al}$ content, around 1 vol.\% of ammonia was added to the lubricant. The surface was then polished by an oxide polishing suspension (OPS) with 2 vol.\% of ammonia and hydrogen peroxide. The specimen was finally electropolished with D2 at $24 \mathrm{~V}$ for $50 \mathrm{~s}$.
The dislocation density was measured using the etch pit technique on the aforescribed electropolished samples, which were additionally etched in 40 wt. $\% \mathrm{HNO}_{3}$ water solution for $10 \mathrm{~s}$ for samples annealed at $750{ }^{\circ} \mathrm{C}$ and $15 \mathrm{~s}$ for samples annealed at $950{ }^{\circ} \mathrm{C}$ and the diffusion couple to produce etch pits. Then, (100)-oriented grains were selected to measure the dislocation density via the etch-pitting technique, since all dislocations intersect the surface for grains oriented in this way [40, 41]. The images of dislocation pits were mainly captured by optical microscope. Atomic force microscopy (AFM, Veeco Dimension 3100) was utilized for pure copper annealed at $750{ }^{\circ} \mathrm{C}$ for $72 \mathrm{~h}$ because of densely arranged dislocations. The area for each image for measuring the dislocation density was $120 \times 120 \mu \mathrm{m}^{2}$. For the diffusion couple, the overall measured area covered the entire diffusion couple [22].

For composition analysis, the diffusion couple was ground and mechanically polished by OPS. The local chemical composition was measured at $15 \mathrm{kV}$ by an electron probe micro-analyzer (EPMA, JEOL JXA-8100). Later, the SFE were calculated from the $\mathrm{Al}$ content from data in the literature $[42,43]$. Four coherent TBs in the diffusion couple were selected for indenting, with the local aluminum content producing SFEs of 78, 40, 34 and $25 \mathrm{~mJ} /$ $\mathrm{mm}^{2}$, estimated from combined results of EBSD and composition analysis [22].

The microstructure of twin boundaries and positions of indents were examined at $15 \mathrm{kV}$ by a scanning electron microscope (SEM, ZEISS Gemini SEM 500) equipped with a 4-quadrant backscatter electron detector (QBSD). Large defects at twin boundaries, such as steps or incoherent twin boundaries, were deliberately avoided during indenting.

Transmission electron microscopy (TEM) foils with $\sim 3 \mathrm{~mm}$ in diameter and $500 \sim 700 \mu \mathrm{m}$ in thickness were cut by EDM from the two pure $\mathrm{Cu}$ samples, and then were mechanically ground to a thickness of $\sim 100 \mu \mathrm{m}$. The samples were electropolished at $-30{ }^{\circ} \mathrm{C}$ using a solution of 30 vol.\% nitric acid and 70 vol.\% methanol at a voltage of $\sim 15 \mathrm{~V}$ in a Struers double-jet electropolisher.

Before each TEM experiment, the specimen was cleaned for $15 \mathrm{~min}$ by argon ion milling at $1.5 \sim 2 \mathrm{keV}$ (Gatan, PIPS II, model 695). The TEM observation was carried out at $200 \mathrm{kV}$ using a Jeol 2200FS. A double-tilt holder was used so that the specimen could be tilted to the right zone axis for Burgers vector analysis, which is determined via the invisibility criterion $\underline{g} \cdot \underline{b}=0$ ( $\underline{g}$ is the diffracting vector and $\underline{b}$ is the Burgers vector). Images were recorded with scanning transmission electron microscopy (STEM).

Nanoindentation tests were performed on $\{110\}$-oriented grains with a Nanoindenter G200 (Agilent Technologies) using two spherical diamond indenters with radii of $\sim 1.5$ and $\sim 1 \mu \mathrm{m}$ provided by Synton MDP (Switzerland). The indenter radii were calibrated by a method developed by Swadener et al. 
$[44,45]$. Constant strain rate loading with $\dot{P} / P=0.05 \mathrm{~s}^{-1}$ was employed in all experiments. The indent spacing was carefully chosen to exclude indent-indent interactions [17].

It is difficult to place nano-indents exactly on $\Sigma 3$ twin boundaries or in close proximity to the boundary for three reasons:

1. The TB must not be visible in the optical microscope of the indenter.

2. The position reproducibility of the nanoindenter stage is $1 \mu \mathrm{m}$.

3. The probability of hitting a boundary is very low if a random array is placed on the sample.

Before describing our strategy to properly hit a boundary, we wish to discuss point (1) in greater detail. Essentially, all optical contrasting mechanisms (e.g., grain boundary grooves, oxide layers, different crystallographic orientations) would violate the assumptions on the Hertzian stress field of the indenter. Our assumptions are no oxide layer, a flat surface, and elastic homogeneity in the compression direction. Thus, as soon as one can contrast the TB by optical microscopy, the assumptions of the indent interpretation are violated.

To hit the boundary, we approached it by inclining a line of indents with respect to the $\mathrm{TB}$ by $\sim 1^{\circ}$. This requires a certain TB length (at least $150 \mu \mathrm{m}$ ) to assure that we cross the boundary with our line of indents. For each line of indents, one to three indents (either the indent center or the contact radius at pop-in load) hits/hit the boundary.

\section{Acknowledgments}

Juan Li would like to thank the China Scholarship Council (CSC) for the scholarship granted to support this work. Her contributions to this work include the experimental data collection, statistical interpretation and manuscript preparation. GMP's contributions to this work, which included conceptualization and design of the nanoindentation experiments, interpretation of results, and assistance in writing the manuscript, were supported by the US Department of Energy, National Nuclear Security Administration under Award no. DE-NA0003857. CK's contributions to this work included conceptualization and design of the nanoindentation experiments, interpretation of results, development of the theoretical model, manuscript writing and supervision. The authors thank Dr. Minjie Lai and Dr. Jiwon Jeong from MPIE for their assistance during TEM investigations.

\section{Funding}

Open Access funding enabled and organized by Projekt DEAL.

\section{Data Availlability}

The datasets generated during and/or analyzed during the current study are available from the corresponding author on reasonable request.

\section{Declarations}

Conflict of interest On behalf of all authors, the corresponding author states that there is no conflict of interest.

\section{Open Access}

This article is licensed under a Creative Commons Attribution 4.0 International License, which permits use, sharing, adaptation, distribution and reproduction in any medium or format, as long as you give appropriate credit to the original author(s) and the source, provide a link to the Creative Commons licence, and indicate if changes were made. The images or other third party material in this article are included in the article's Creative Commons licence, unless indicated otherwise in a credit line to the material. If material is not included in the article's Creative Commons licence and your intended use is not permitted by statutory regulation or exceeds the permitted use, you will need to obtain permission directly from the copyright holder. To view a copy of this licence, visit http://creativecommons.org/ licenses/by/4.0/.

\section{Supplementary Information}

The online version contains supplementary material available at https://doi.org/10.1557/s43578-021-00253-y.

\section{References}

1. Z. Shen, R. Wagoner, W. Clark, Scr. Metall. 20(6), 921 (1986)

2. Z. Shen, R. Wagoner, W. Clark, Acta Metall. 36(12), 3231 (1988)

3. J.C. Li, Trans. Metall. Soc. AIME 227(1), 239 (1963)

4. L. Murr, Mater. Sci. Eng. 51(1), 71 (1981)

5. D.C. Bufford, Y.M. Wang, Y. Liu, L. Lu, MRS Bull. 41(4), 286 (2016)

6. L. Lu, X. Chen, X. Huang, K. Lu, Science 323(5914), 607 (2009)

7. X. Li, Y. Wei, L. Lu, K. Lu, H. Gao, Nature 464(7290), 877 (2010) 
8. L. Lu, Y. Shen, X. Chen, L. Qian, K. Lu, Science 304(5669), 422 (2004)

9. Y.M. Wang, F. Sansoz, T. LaGrange, R.T. Ott, J. Marian, T.W. Barbee Jr., A.V. Hamza, Nat. Mater. 12(8), 697 (2013)

10. J.R. Morris, H. Bei, G.M. Pharr, E.P. George, Phys. Rev. Lett. 106(16), 165502 (2011)

11. C. Begau, A. Hartmaier, E.P. George, G.M. Pharr, Acta Mater. 59(3), 934 (2011)

12. D. Lorenz, A. Zeckzer, U. Hilpert, P. Grau, H. Johansen, H. Leipner, Phys. Rev. B. 67(17), 172101 (2003)

13. D. Bahr, G. Vasquez, J. Mater. Res. 20(08), 1947 (2005)

14. D. Bahr, D. Kramer, W. Gerberich, Acta Mater. 46(10), 3605 (1998)

15. T. Li, Y. Gao, H. Bei, E.P. George, J. Mech. Phys. Solids. 59(6), $1147(2011)$

16. A.C. Lund, A.M. Hodge, C.A. Schuh, Appl. Phys. Lett. 85(8), $1362(2004)$

17. J. Li, G. Dehm, C. Kirchlechner, Materialia. 7, 100378 (2019)

18. H. Bei, Y.Z. Xia, R.I. Barabash, Y.F. Gao, Scr. Mater. 110, 48 (2016)

19. A. Barnoush, H. Vehoff, Acta Mater. 58(16), 5274 (2010)

20. E. Nes, Acta Metall. Mater. 43(6), 2189 (1995)

21. T. Britton, D. Randman, A. Wilkinson, J. Mater. Res. 24(3), 607 (2009)

22. J. Li, C. Kirchlechner, Mater. Charact. 110136 (2020)

23. S. Pfeiffer, M.F.-X. Wagner, Proc. R. Soc. A 473(2204), 20170330 (2017)

24. H. Gleiter, Acta Metall. 17(12), 1421 (1969)

25. J. Hirth, R. Balluffi, Acta Metall. 21(7), 929 (1973)

26. R.E. Smallman, A.H.W. Ngan, Introduction to Dislocations, in Modern Physical Metallurgy (Eighth Edition), edited by R. E. Smallman and A. H. W. Ngan (Butterworth-Heinemann, City, 2014), pp. 121.
27. Z.Y. Liang, J.T.M. De Hosson, M.X. Huang, Acta Mater. 129, 1 (2017)

28. K. Westmacott, R. Barnes, D. Hull, R. Smallman, Philos. Mag. 6(67), 929 (1961)

29. J. Silcox, P. Hirsch, Philos. Mag. 4(37), 72 (1959)

30. J. Hirth, J. Lothe, Theory of Dislocations, vol. Second (Krieger Publishing Company, Malabar, 1982).

31. D. Bufford, Y. Liu, J. Wang, H. Wang, X. Zhang, Nat. Commun. $\mathbf{5}(1), 1(2014)$

32. Y. Wang, M. Sui, E. Ma, Philos. Mag. Lett. 87(12), 935 (2007)

33. M. Sennour, S. Lartigue-Korinek, Y. Champion, M. Hÿtch, Philos. Mag. 87(10), 1465 (2007)

34. J. Wang, N. Li, O. Anderoglu, X. Zhang, A. Misra, J. Huang, J. Hirth, Acta Mater. 58(6), 2262 (2010)

35. C.J. Shute, B. Myers, S. Xie, S.-Y. Li, T. Barbee Jr., A. Hodge, J. Weertman, Acta Mater. 59(11), 4569 (2011)

36. M.A. Meyers, K.K. Chawla, Mechanical Behavior of Materials (Cambridge University Press, Cambridge, 2008).

37. H. Van Swygenhoven, P. Derlet, A. Hasnaoui, Phys. Rev. B. 66(2), 024101 (2002)

38. H. Van Swygenhoven, P. Derlet, A. Frøseth, Acta Mater. 54(7), 1975 (2006)

39. D.E. Spearot, K.I. Jacob, D.L. McDowell, Acta Mater. 53(13), 3579 (2005)

40. F. Young Jr., J. Appl. Phys. 32(2), 192 (1961)

41. A. Ruff Jr., J. Appl. Phys. 33(12), 3392 (1962)

42. A. Rohatgi, K.S. Vecchio, G.T. Gray III., Metall. Mater. Trans. A. 32(1), 135 (2001)

43. C. Huang, W. Hu, G. Yang, Z. Zhang, S. Wu, Q. Wang, G. Gottstein, Mater. Sci. Eng., A 556, 638 (2012)

44. J.G. Swadener and G.M. Pharr, MRS Online Proceedings Library Archive. 594, (1999).

45. W. Li, H. Bei, J. Qu, Y. Gao, J. Mater. Res. 28(14), 1903 (2013) 